\title{
Secondary pharmacology data to assess potential off-target activity of new drugs: a regulatory perspective
}

\author{
Thomas Papoian, Haw-Jyh Chiu, Ikram Elayan, Gowraganahalli Jagadeesh, \\ Imran Khan, Adebayo A. Laniyonu, Cindy Xinguang Li, Muriel Saulnier, \\ Natalie Simpson and Baichun Yang
}

Secondary pharmacology studies evaluate compounds for pharmacodynamic activity against a broad range of targets that are related to or distinct from the intended therapeutic target. This is a cost-effective approach used by many pharmaceutical companies as a safety screen early in drug development, as highlighted in a recent article (Reducing safety-related drug attrition: the use of in vitro pharmacological profiling. Nature Rev. Drug Discov. 11, 909-922 (2012)) $)^{1}$. Information on the potency of a drug for a given biological target can be used to determine structure-activity relationships, assess potential liability for off-target effects, and influence early clinical trial design, dose selection and patient monitoring.

Although secondary pharmacology data are often included early in the drug submission process as part of a standard safety pharmacology screen ${ }^{2}$, there is variability in the timing, type, extent and format of secondary pharmacology data submitted to regulatory agencies ${ }^{1}$. This Correspondence addresses these issues by discussing the utility of and regulatory perspectives on in vitro secondary pharmacology data, how such data are used in the Center for Drug Evaluation and Research at the US Food and Drug Administration and when such data are usually submitted. We hope that such a discussion could be helpful for drug developers when considering the type and extent of secondary pharmacology data to submit to regulatory agencies in support of a drug application.

\section{Data submission and use Useful aspects of submitted secondary pharmacology data. Detailed aspects of secondary pharmacology data that are con- sidered to be useful during regulatory safety assessments are summarized in TABLE 1. Incorporation of these aspects into secondary pharmacology study reports not only enhances the quality and regulatory utility of the reports but also decreases uncertainty in decision making owing to misinterpretation of data.}

Regulatory assessment of in vitro secondary pharmacology data. Various protocols for secondary pharmacology studies are commonly available and generally consist of binding assays, functional assays and enzyme assays, all of which provide important information regarding the pharmacological activity of a drug and possible unanticipated side effects that may be seen in humans. When the data suggest possible off-target activity, the extent of that risk under clinically relevant conditions and drug exposures is carefully assessed, and safety recommendations are made for the proposed clinical studies or additional non-clinical studies are requested to help to better understand or reduce the risk.

In our experience, the panels of targets that are employed vary widely and are often selected without justification or a description of their relevance to human safety. In such cases, a repeat of the study may be recommended using a more appropriate selection of clinically relevant targets. It is useful to determine whether a drug shares similar off-target activity with other drugs in the class - for example, the sedative and anticholinergic properties of certain histamine $\mathrm{H}_{1}$ receptor antagonists - so that such side effects in humans can be better anticipated and monitored. Similarly, target panel selection may also include consideration of whether the intended drug target has structural or functional similarity to a related but unintended target. If such a relationship is found, then clinical consequences of affecting those targets are considered. Drugs may also possess specificity for, or a mechanism

\footnotetext{
Table 1 | Aspects of secondary pharmacology data considered useful for regulatory review

\begin{tabular}{|c|c|}
\hline Aspect & Description \\
\hline Target panel & $\begin{array}{l}\text { - For new molecular entities, a broad panel of } G \text { protein-coupled receptors, transporters, ion channels, nuclear receptors and } \\
\text { enzymes, including various kinases } \\
\text { - Safety or pharmacological rationale provided for selection of targets } \\
\text { - Panel of targets not limited to the same drug class, putative mechanism of action or group of related chemical structures as } \\
\text { the lead compound }\end{array}$ \\
\hline $\begin{array}{l}\text { Methodology } \\
\text { section }\end{array}$ & $\begin{array}{l}\text { - Detailed description of methodology used for all binding and functional assays } \\
\text { - Rationale and/or criteria for a positive response (for example, } \geq 50 \% \text { inhibition or stimulation at the maximum concentration tested) }\end{array}$ \\
\hline $\begin{array}{l}\text { Discussion } \\
\text { section }\end{array}$ & $\begin{array}{l}\text { - Biological significance of findings } \\
\text { - How potency of off-target activity compares with that of the intended target } \\
\text { - Relative in vitro potency for off-target activity versus potency for intended target (or targets) } \\
\text { - IC } C_{50} \text { or } K_{\mathrm{i}} \text { values versus projected or observed plasma drug levels achieved at efficacious doses (animals or humans) } \\
\text { - Correlation between in vitro findings and those observed in animals } \\
\text { - Discussion of possible drug-related effects that should be monitored in humans }\end{array}$ \\
\hline
\end{tabular}
}


of action linked to, selected targets from a large family of related targets that have structural or functional similarity (for example, the ATP-binding site of protein tyrosine kinases). In such cases, a broad panel screen of all closely related family members may be useful in distinguishing intended pharmacological effects from unintended effects.

It is important to understand how findings relate to those observed with other members of the drug class or for a certain mechanism of action. If the findings are similar, then the clinical risk may be considered to be similar as well. If they are different, however, then further evaluation, such as follow-up in vitro or animal studies focusing on the different targets affected, may be recommended to better define the risk. Correlations between positive secondary pharmacology results and results seen in animals may be used to determine whether in vitro activity translates to possible human risk under therapeutic conditions. It is important to note that in vitro panels generally use human-specific targets, and in vitro results may not always correlate with those observed in animals. Correlations between in vitro half-maximal inhibitory concentration $\left(\mathrm{IC}_{50}\right)$ or inhibition constant $\left(K_{\mathrm{i}}\right)$ values with in vivo plasma drug levels are also used to assess possible effects at therapeutic exposures. The closer the $\mathrm{IC}_{50}$ or $K_{\mathrm{i}}$ values are to plasma drug levels, the more likely the clinical effect. Also considered in this context are local tissue drug concentrations at the site of action that may be higher than circulating levels. Use of focused profiling may be recommended to address specific issues of concern, such as the safety of active drug metabolites or unique major human metabolites not formed in animals but found during subsequent clinical testing. Even if a compound produces a positive in vitro result at therapeutically

\section{Table 2 | Examples of regulatory actions based on positive off-target activity}

\section{Case example}

Endocrine disruptors

Peroxisome proliferatoractivated receptor- $\gamma$ agonists (for example, thiazolidinediones)

Cardiac (voltage-gated) ion channel protein antagonists

5- $\mathrm{HT}_{2 \mathrm{~B}}$ (serotonin) receptor agonists (for example, fenfluramine-phentermine, 3,4-methylenedioxy- $\mathrm{N}$ methylamphetamine (MDMA; also known as ecstasy)

Ligand-gated ion channels: $\mathrm{N}$-methyl-D-aspartate (NMDA) receptor antagonists (for example, dizocilpine (MK-801) and ketamine)

Drugs with potential for abuse

\section{Drug effects}

- Interference with some aspect of the endocrine system

- Blocking of hormone receptors (membrane and nuclear)

- Effects on synthesis, transport or excretion of hormones

- Sex hormones (oestrogens and androgens) most commonly affected

- Can affect development and maturation

Human target organs include heart (in the case of congestive heart failure), kidney (fluid retention), skeletal muscle (rhabdomyolysis), and liver, bone marrow and bladder (cancers)

\section{Possible regulatory action}

- Use of receptor-binding and enzyme assays to screen for potential unintended effects, including interference with certain endocrine receptors

- If in vitro results are positive, additional studies may be warranted:

Developmental and reproductive toxicity, pre- and post-natal development, and juvenile animal toxicity studies to assess endocrine-related developmental effects;

Assessment of hormone levels in human clinical trials

- Significant off-target activity in vitro (for example, low effector concentration for half-maximum response $\left(\mathrm{EC}_{50}\right)$ in cell-based reporter gene assay) may result in:

Additional monitoring to assess human risk at therapeutic drug concentrations;

Rodent carcinogenicity studies to support clinical studies of $>6$ months duration

- Potassium voltage-gated channel subfamily $\mathrm{H}$ member 2 Positive results may prompt additional (hERG1), which mediates the repolarizing current $I_{\mathrm{kr}}$ : Antagonists slow conduction and repolarization of action potential;

Associated with corrected QT prolongation and torsades de pointes

- Sodium channel protein type 5 subunit-a (Nav1.5):

Antagonists prolong QRS interval and slow conduction

\section{electrocardiography monitoring in patients}

Agonist activity to $5-\mathrm{HT}_{2 \mathrm{~B}}$ receptors on heart valve leaflets known to result in fibrotic cardiac valvulopathy

- In vitro agonist activity (for example, calcium flux assay) may result in:

Request for focused histopathology of animal heart valves;

Additional cardiac monitoring (echocardiography) in humans if the safety margin is sufficient based on the animal no-observable adverse effect level

- If no safety margin, then possible clinical hold due to serious and irreversible nature of the effect

- Protect neurons against excess glutamate neurotoxicity - Used to treat stroke, epilepsy, pain and Parkinson disease

- Can injure or destroy certain other neurons and induce psychotic symptoms and memory impairment

- Mechanism of toxicity is complex, involving cholinergic, glutamatergic, GABAergic, dopaminergic and/or noradrenergic systems

Off-target activity affecting the neurotransmitter system, (for example, dopamine, noradrenaline, serotonin, GABA ( $\gamma$-aminobutyric acid)), acetylcholine, opioid (for example, $\mu$-type), NMDA or cannabinoid receptors)
Positive in vitro results may prompt focused animal studies for possible neurotoxic effects on structure (expanded neurohistopathology) and function (neurobehavioural testing, including evaluation for effects on sensory, motor and cognitive functions)

- Positive results may prompt:

Further animal and human behavioural studies for addictive or abuse liability;

Drug scheduling by the US Drug Enforcement Administration 
relevant concentrations, certain risk-benefit considerations for particular patient populations are taken into account, such as the seriousness of the therapeutic indication and whether potential adverse effects are considered to be reversible.

Case examples of regulatory actions based on positive off-target activity are provided in TABLE 2 and include endocrine disruptors, peroxisome proliferator-activated receptor- $\gamma$ agonists, cardiac (voltage-gated) ion channel protein antagonists, $5-\mathrm{HT}_{2 \mathrm{~B}}$ (serotonin) receptor agonists, ligand-gated ion channel protein (for example, $N$-methyl$\mathrm{D}$-aspartate receptor) antagonists and drugs with potential for abuse. Although not exhaustive, these examples highlight current experience with certain classes and actions of drugs that have resulted in specific regulatory actions based on their potential risk for human safety.

\section{Timing of secondary pharmacology data} submission. For proper safety assessment, it is advantageous for secondary pharmacology data to be available with the initial submission to support first-in-human (Phase I) clinical trials. Such data can also be used to help to interpret clinical findings or adverse drug reactions that occur as drug development proceeds into later phases of clinical testing.

\section{Summary and conclusions}

In vitro secondary pharmacology studies are considered to be valuable and cost-effective tools to identify and to predict possible adverse effects of drugs in early human clinical trials based on unintended pharmacodynamic activities. For new molecular entities, there is a general preference for a broad panel of targets. Also useful are detailed descriptions for methodology, data expression in tabular and/or graphical format and discussion of the biological significance of any findings. When used with other standard non-clinical assessments, such data help to reduce drug attrition due to safety concerns and increase the chances that safe and effective therapeutics will be made available to the public.

Variability in the timing, type, extent and format of submitted secondary pharmacology data limits the usefulness of the data during early stages of the drug safety review process. This Correspondence addresses these issues by providing current regulatory perspectives on important aspects of in vitro secondary pharmacology data, as well as discussing how such data are assessed and when such data are usually submitted.

Important remaining issues are identifying specific targets, target categories and biological functionalities that should constitute a minimal in vitro panel, and determining how primary versus secondary panels should be defined. Although some of the more general considerations involved in target selection are discussed here, several recent publications are available that provide additional rationale for target selection under specific circumstances $^{3-10}$.

Thomas Papoian, Gowraganahalli Jagadeesh, Muriel Saulnier and Baichun Yang are at the Division of Cardiovascular and Renal Products, US Food and Drug Administration.

Haw-Jyh Chiu and Natalie Simpson are at the Division of Hematology Oncology Toxicology, US Food and Drug Administration.
Ikram Elayan and Imran Khan are at the Division of Psychiatry Products,

US Food and Drug Administration.

Adebayo A. Laniyonu is at the Division of Medical Imaging Products, US Food and Drug Administration.

Cindy Xinguang Li is at the Division of Nonprescription Drug Products, US Food and Drug Administration, 10903 New Hampshire Avenue, Silver Spring, Maryland 20993, USA.

Correspondence to T.P. e-mail:Thomas.Papoian@fda.hhs.gov doi: 10.1038/nrd3845-c1

1. Bowes, J. et al. Reducing safety-related drug attrition: the use of in vitro pharmacological profiling. Nature Rev. Drug Discov. 11, 909-922 (2012).

2. ICH Expert Working Group. Safety pharmacology studies for human pharmaceuticals S7A. ICH [online], http://www.ich.org/fileadmin/Public Web_Site/ICH Products/Guidelines/Safety/S7A/Step4/S7A Guideline. pdf (2001).

3. Bass, A. S. et al. Exploratory drug safety: a discovery strategy to reduce attrition in development. J. Pharmacol. Toxicol. Methods 60, 69-78 (2009).

4. Hughes, J. P. et al. Principles of early drug discovery. Br. J. Pharmacol. 162, 1239-1249 (2011).

5. Lounkine, E. et al. Large-scale prediction and testing of drug activity on side-effect targets. Nature 486, 361-367 (2012).

6. Muller, P. Y. \& Milton, M. N. The determination and interpretation of the therapeutic index in drug development. Nature Rev. Drug Discov. 11, 751-761 (2012).

7. Redfern, W. S. et al. Safety pharmacology a progressive approach. Fundam. Clin. Pharmacol. 16, 161-173 (2002)

8. Valentin, J. P. \& Hammond, T. Safety and secondary pharmacology: successes, threats, challenges and opportunities. J. Pharmacol. Toxicol. Methods 58, 77-87 (2008).

9. Wakefield, I. D. et al. The application of in vitro methods to safety pharmacology. Fundam. Clin. Pharmacol. 16, 209-218 (2002).

10. Whitebread, S. et al. In vitro safety pharmacology profiling: an essential tool for successful drug development. Drug Discov. Today 10, 1421-1433 (2005).

Disclaimer

This article reflects the views of the authors and should not be construed as representing views or policies of the US Food and Drug Administration.

Competing interests statement

The authors declare no competing interests. 